\title{
EVALCATION OF A COMPUTATIONAL MODEL OF SITUATIONAL AWARENESS
}

\author{
Mark D. Burdick \\ Siln Jose State I niversity \\ mburdickerimail.arc.nasa.gov
}

\author{
R. Iay Shively \\ Acroflightdynamics Dircetorate (AMRDF: \\ US Army Aviation and Missile Command \\ jshively@mail.arc.nasa.gov
}

Army/NASA Rotorcraft Division

Ames Research Center

Moffett Ficld, CA

\begin{abstract}
Although the use of the psychological construct of situational awareness (SA) assists researchers in creating a flight environment that is safer and more predictable, its true potential remains untapped until a valid means of predicting SA a priori becomes available. Previous work proposed a computational model of SA (CSA) that sought to fill that void. The current line of research is aimed at validating that model. The results show that the model accurately predicted $\mathrm{SA}$ in a piloted simulation.
\end{abstract}

\section{INTRODUCTION}

\section{Computation Model of Situational Awareness}

In an effort to predict the potential impact on situational awareness (SA) of different tasks, designs and environments, Shively, Brickner \& Silbiger (1997) created a computational model of situational awareness (CSA). Initially designed as a feature of the Man-machine Integration Design and Analysis System (MIDAS) (Smith \& Tyler, 1997), the CSA, at its simplest, is the ratio of the operator's relevant knowledge to the information needed for the mission task.

One original facet of the CSA was the differentiation between perceived, actual and crroneous SA. Perceived SA is what operators work with, the level of $S A$ they think they have. Iowever, it may include errors in perception or identilication. Erroneous SA represents situational elements (SE's) that are misperceived or misidentified. Actual SA differs from pereeived SA by including information that is otherwise unknown to the operator less the error component.

\section{Goals of Research}

The goals of this research were to: (1) Continue to reline a clearly delined, computational, prediclive model of $\mathrm{S} \wedge,(2)$ Distinguish between perceived and actual SA, and (3) Compare the predictions of this model to measured levels of SA in the context of piloted simulation.

\section{Situational Awareness Model}

The current CSA model in MIDAS is comprised of two essential features: situational elements and situation-specific nodes. These two features will be described briefly, but for a more detailed description of the model see Shively et al., (1997).

Situational elements (SE's) are relevant information in the environment that define the circumstances. These include such things as other aircraft, obstacles, waypoints, ownship parameters, etc. The pilot experiences these elements through perception, experience or a pre-flight briefing. Each SE has a mathematical weight attached to it based upon its importance in the situation. In addition to its weight, each SE has a mathematical value based upon one of four levels of awareness. These four levels of awareness (detection, recognition, identification and comprehension) provide a means of quantifying an operator's perception of SE.

Situation-sensitive nodes are semantically related collections of SF:'s. The nodes are defined by what is important in the context of a given task and are weighted by the overall importance of the 
moke in ketermining the level of St. Thes, in the current study; narigation represented one of the nodes. It was comprised of sil:'s concerning course'.

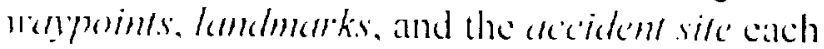
pilot wats trying to reach. Because visual navigation was so important to the task, the weight on the node was 0.5 . If the situation had changed then the weights on the nodes, or the nodes themselves, might have changed to reflect the ideal $S A$ accurately.

\section{COURSE OF RESEARCH}

A series of three studies, each a more stringent test of the predictions of the model, were conducted at NASA Ames Research Center. Each study sought to validate the CSA by comparing its predictions of pilot SA to subjective and objective measures of SA using generally accepted measurement techniques in the context of a civil helicopter mission. Burdick and Shively (1999) reported pertinent details of the initial two studies. This paper will focus on the third.

1

\section{The Rotorcraft Part-Task Laboratory (RPTL)}

The goal of the current study was to continue the experimental validation of the SA model created by Shively, Brickner and Silbiger. This was accomplished by creating a family of scenarios, which differed only in the level of awareness of the SE's. These scenarios generated low, medium and high levels of predicted SA by the model. Predicted SA levels were compared to scores from a battery of accepted SA measures such as the, Situational Awareness Rating Technique (SART, Taylor, 1989) and the Situational Awareness Global Assessment Technique (SAGAT, Endsley, 1995). Again, the goal was to determine how well the model was able to predict mathematically the empirical measures of $\mathrm{SA}$.

\section{METHOD}

\section{Participants and Materials}

Six general aviation pilots were trained in the Rotoreralt Part-Task Laboratory (RPTI, at NASA Ames Rescarch Center. This single-person, lixed-hase simulater consists of an out-the-window view and an instrumentation pand; aach displated on a Silicon Ciraphics Inc. (S(i) monitor. The simulator rotorcralt dynamics were generaled by the Finhanced Stability Derivative (ISSD) model (Whalley, 1994) and pilot control was through a "flybox" consisting of a lever representing the collective and a two-axis joystick that controlled pitch, roll and yaw. Figures $I$ and 2 display the out-the-window view and the instrumentation panel, respectively.

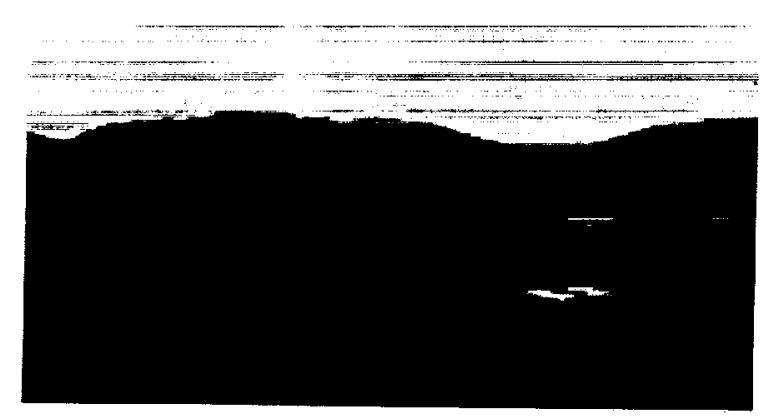

Figure 1. Out-the-window view

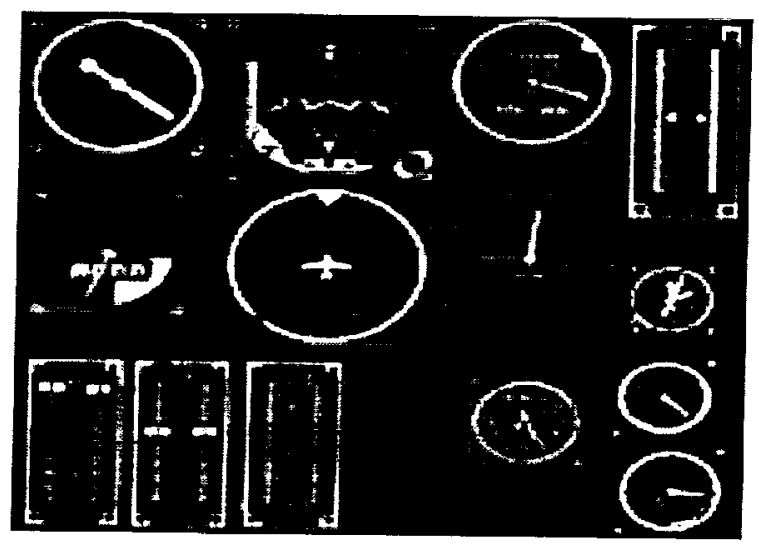

Figure 2. Flight Instrumentation

\section{Design}

The study was based on a one-way (predicted high, medium or low SA) withinsubjects design. Each pilot completed nine Medical Evacuation (MEDEVAC) scenarios consisting of a flight to an accident site followed by a patienttransport segment toward a pre-selecked hospital or airport. Fach seenario was repeated three times with different levels of information available to the pilots, creating the three predicted levels of SA. 
mok in dekmining the kevel of St. Thus, in the current study; nerigation represented one of the nodes. It was comprised of slit's concerning comrice, wapoints, lamdmorks, and the acident site each pilot was trying to reach. Beciuse visual navigation was so important to the task, the weight on the node was 0.5 . If the situation had changed then the weights on the nodes, or the nodes themselves, might have changed to reflect the ideal $S A$ accurately.

\section{COURSE OF RESEARCH}

A series of three studies, each a more stringent test of the predictions of the model, were conducted at NASA Ames Research Center. Each study sought to validate the CSA by comparing its predictions of pilot SA to subjective and objective measures of SA using generally accepted measurement techniques in the context of a civil helicopter mission. Burdick and Shively (1999) reported pertinent details of the initial two studies. This paper will focus on the third.

\section{The Rotorcraft Part-Task Laboratory (RPTL)}

The goal of the current study was to continue the experimental validation of the SA model created by Shively, Brickner and Silbiger. This was accomplished by creating a family of scenarios, which differed only in the level of awareness of the SE's. These scenarios generated low, medium and high levels of predicted SA by the model. Predicted SA levels were compared to scores from a battery of accepted SA measures such as the, Situational Awareness Rating Technique (SART, Taylor, 1989) and the Situational Awareness Global Assessment Technique (SAGAT, Endsley, 1995). Again, the goal was to determine how well the model was able to predict mathematically the empirical measures of $\mathrm{SA}$.

\section{METHOD}

\section{Participants and Materials}

Six general aviation pilots were trained in the Rotorcraft Part-Task Laboratory (RPTI.) at NASA Ames Research Center. This single-person, lixed-base simulator consists of an out-the-window view and an instrumentation panel; each displiyed on a Silicon (iraphics Inc. (SGI) monitor. The simulator rotorcralt dynamics were generaled by the Enhanced Stability Derivative (ISSD) model (Whalley, 1994) and pilot control was through a "flybox" consisting of a lever representing the collective and a two-axis joystick that controlled pitch, roll and yaw. Figures 1 and 2 display the out-the-window view and the instrumentation panel, respectively.

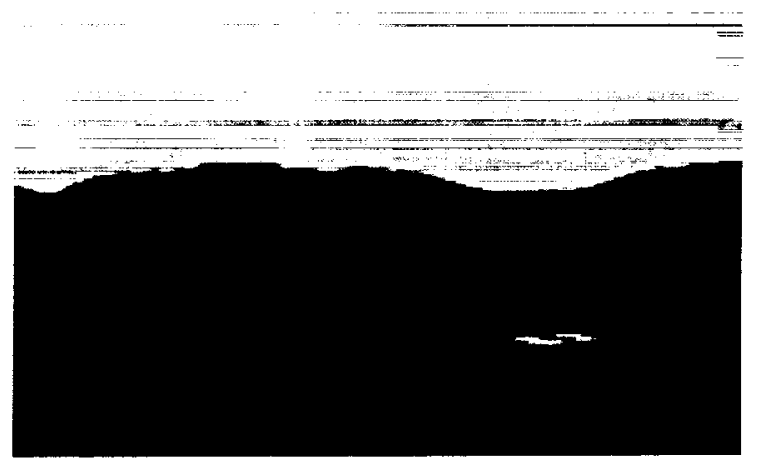

Figure 1. Out-the-window view

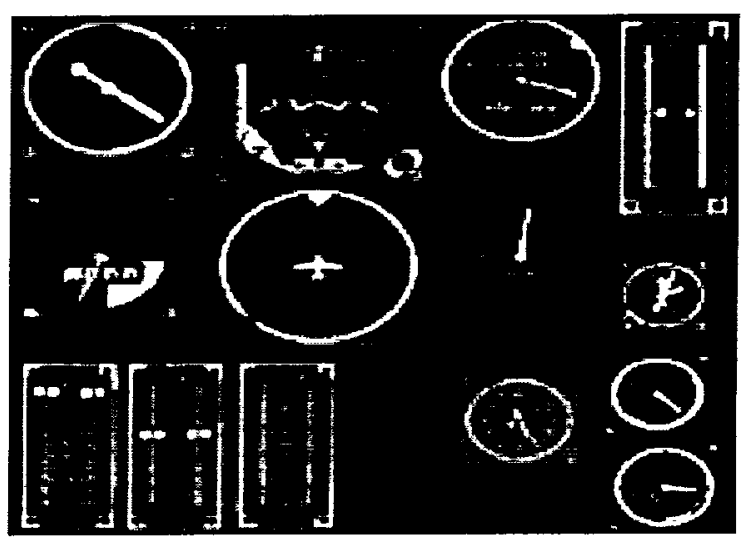

Figure 2. Flight Instrumentation

\section{Design}

The study was based on a one-way (predicted high, medium or low SA) withinsubjects design. Each pilot completed nine Medical Evacuation (MEDEVAC) scenarios consisting of a flight to an accident site followed by a patienttransport segment toward a pre-selected hospital or airport. Each scenario was repeated three times with different levels of information available to the pilots, creating the three predicted levels of $\mathrm{S} \Lambda$. 
A scomario lask analysis, performed in conjunction with experienced MEIDI:VAC pilot input. determined the relevant situational elements for the task. These situational elements were amalyzed and aggregated into three situationspecilic nodes that were then weighted based on their importance in the scenario. Table I indicates the three nodes along with the computational weights and SE's associated with each node.

Table 1. Situation-specific nodes, weights and situational elements.

\begin{tabular}{|c|c|c|}
\hline Node & Weight & Situational Elements \\
\hline Navigation & 0.5 & $\begin{array}{c}\text { Briefed course, heading, } \\
\text { waypoints, landmarks, } \\
\text { accident site }\end{array}$ \\
\hline Ownship & 0.3 & $\begin{array}{c}\text { Airspeed, radar altitude, } \\
\text { V/S, fuel quantity and } \\
\text { consumption, engine and } \\
\text { oil temps, percent torque }\end{array}$ \\
\hline Air Traffic & 0.2 & $\begin{array}{c}\text { Visible traffic bearing, } \\
\text { heading, relative altitude }\end{array}$ \\
\hline
\end{tabular}

The three experimental conditions of predicted high, medium or low SA were created by manipulating the level of awareness of the various situational elements in the task. For example, in the conditions predicted to lead to high SA the situational element "briefed course" was a clearly defined course on a map given to the pilots. In the conditions predicted to lead to medium levels of $S A$, the SE was a lightly scribed course on the map. For the conditions predicted to lead to low SA the SE was a map with no obvious course inscribed on it.

After manipulation, all SE's were assigned mathematical weights based on the level of awareness of the SE in each trial. Arbitrary values o $0.20,0.50,0.75$, and 1.00 were assigned to the four levels of awareness (e.g. Detection, Recognition, Identification and Comprehension), respectively. These linear values were chosen arbitrarily and are subject to change, pending results of validation efforts. For a complete listing of manipulations and example of the mathematical computation, see Burdick \& Shively (1999).

\section{Procedure}

Pilots partic

conseculive days.

required two hours 6.

er

training was conducted to,

the

second session.

Before each experimental sce.

preflight briefing was conducted that descr sed the route, waypoints, landmarks, headings, time between waypoints and total time. Additionally, each pilot was given an enlarged sectional map of the flight area along with a set of text instructions detailing route specifics. Pilots were instructed to fly with an airspeed of 80 to 120 knots and at an altitude of 200 to 400 feet above ground level. Moreover, they were to verbally contact the experimenter when they saw any other air traffic during the flight and relate the aircraft type, relative bearing, heading and relative altitude.

Pilots were briefed that at some point during each leg of the mission, the simulation would pause and SAGAT probes would appear on the Instrumentation Panel. Verbal responses were required for each probe and recorded by the experimenter. After each leg of the flight, pilots received a four-dimensional SART survey, followed by a post-trial questionnaire.

\section{RESULTS}

Three performance measures were calculated to indicate task difficulty between the predicted high, medium and low SA conditions. Table 2 displays the mean altitude, airspeed and course deviation across conditions. As expected, neither airspeed nor course deviation differed significantly across trials. However, contrary to expectations, in the predicted low SA condition pilots tended to fly lower to the ground. This may be due to the reduced visibility that obscured the field of view; pilots flew closer to the ground simply to ensure they saw the landmarks needed for navigation.

Table 2. Mean allitude, airspeed and deviation from course as a function of condition 


\begin{tabular}{|c|c|c|c|}
\hline $\begin{array}{l}\text { Predicted } \\
\mathrm{SA}\end{array}$ & $\begin{array}{c}\text { Alibule } \\
\text { li }^{*}\end{array}$ & $\begin{array}{c}\text { Airspocd } \\
\text { kts }\end{array}$ & $\begin{array}{l}\text { Conurse } \\
\text { deviation } \\
\mathrm{m}\end{array}$ \\
\hline I.ow & 326 & $97.9^{-}$ & 467 \\
\hline $\mathrm{Med}$ & 375 & 96.16 & 475 \\
\hline lligh & 377 & 96.26 & 322 \\
\hline
\end{tabular}

* indicates a significant difference at $p<.05$

Figure 3 shows the SA component of the SART survey compared to the model's prediction of the perceived SA across experimental conditions.

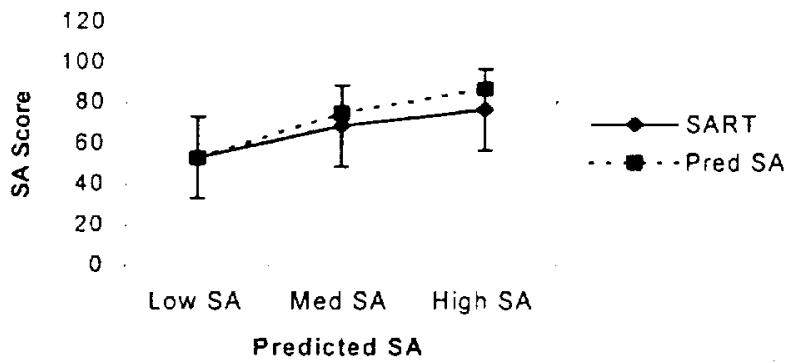

Figure 3. SART SA and predicted Perceived SA as a function of condition

Figure 4 shows two measures taken from the SAGAT probes administered on each half of every trial. The heading error represents the difference between the ownship's true heading and SAGAT response while the accident site heading error is the difference between the direct bearing to the site and that reported by the pilot.

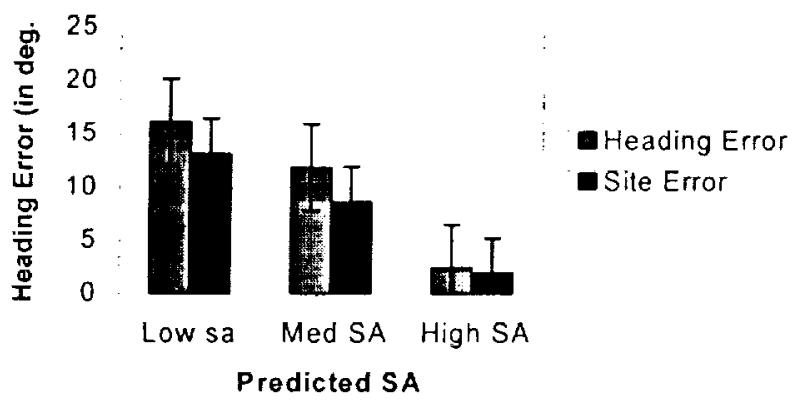

Figure 4. SAGAT Ownship heading and Accident site heading errors

This data shows that pilots in the predicted high $S A$ conditions were significantly more precise in stating not only their ownship's current heading, but were more accurate in giving a bearing to the accident site also.
Further di

$S \wedge G \wedge T$ probes

significantly lo

conditions, they w.

$301^{\circ}$

their altitude in the prea.

10n.

ligure 5 displays the mean $a_{1 .}$ sen

pilot reported and actual altitude aue sund level.

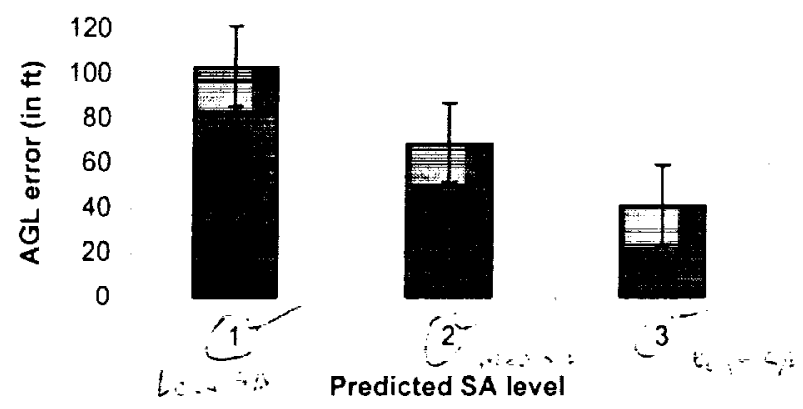

Figure 5. SAGAT AGL Error

predicting

conditions

model usin

flight comr

control of $t$

insertion of

Furthermor

informatior

of perceiver

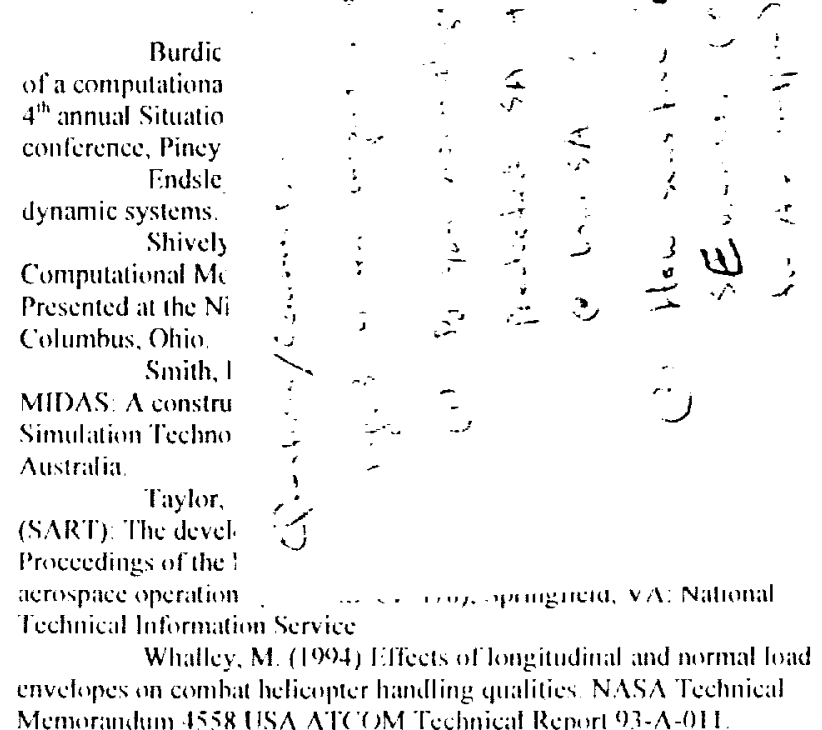

